\title{
Texture profile analyses in tench (Tinca tinca L., 1758) from extensive and intensive culture
}

\author{
František Vácha, Vlastimil Stejskal, Pavel Vejsada, Jan Kouřil, David Hlaváč \\ University of South Bohemia in České Budějovice, Faculty of Fisheries and Protection of Waters, \\ South Bohemian Research Center of Aquaculture and Biodiversity of Hydrocenoses, Institute of Aquaculture, \\ České Budějovice, Czech Republic
}

Received May 29, 2013

Accepted September 26, 2013

\begin{abstract}
The aim of the study was to evaluate the differences in texture profile of tench flesh. Texture profile analyses in tench, two years old, (Tinca tinca L.) was investigated using instrumental texture profile method, focused on hardness, springiness, cohesiveness, gumminess and chewiness performed with Texture Analyser TA.XTPlus. One group of fish was raised extensively in natural earth pond conditions; the other group was intensively cultured in a recirculation system, feeding on a commercial diet for 7 months. Twelve fish ( 6 females and 6 males) from each group were used for analyses. Flesh of male tench in both groups was harder, more gummy and chewy, compared to female. Springiness was lower in tench male from intensive culture and cohesiveness lower in females from both groups. The result of texture analysis in the extensive culture group was 16.01 $\mathrm{N}$ for hardness and $0.72,0.66,10.73$ and 7.69 for springiness, cohesiveness, gumminess and chewiness, respectively. In the intensive culture group it was $15.16 \mathrm{~N}$ for hardness and 0.59 , $0.52,8.06$ and 4.96 for springiness, cohesiveness, gumminess and chewiness, respectively. The results proved that the flesh of fish raised extensively is harder, springier, more cohesive and more gummy, and thus more appealing to consumers. This is the first similar study of texture profile of tench.
\end{abstract}

Cohesiveness, hardness, muscle, springiness, flesh quality

Textural quality is primarily a sensory attribute that is quantified in foods by several instrumental methods. According to Bourne and Szczesniak (2003) and Cardoso et al. (2009), texture profile analysis (TPA) is a resulting force-generated time curve used to quantify a number of textural properties that correlate well with the results from sensory evaluation.

Post mortem textural changes are caused directly or indirectly by physicochemical changes in myofibrillar proteins (Kř́ižek et al. 2011) and changes in extracellular structure such as the loss of fibre compaction and the increase of extracellular space between fibres (Ingolfsdóttir 1997; Popelka et al. 2012). Muscle texture is also affected by other variables such as fish rearing, seasonality and methods of capture, handling and processing (Johnston et al. 2000; Periago et al. 2005). The main quality indicators for fresh fish are fat, colour, texture and freshness. Other indices commonly cited are white stripes (connective tissue), bloodstains, marbling and melanin.

Double compression used in the instrumental procedure enables performing TPA as a plot of force-time curves (Rahman 2006). Other terms describing texture are firmness, stiffness and the yield point (Vácha et al. 2007). Many attempts have been made to correlate physical measurements with sensory evaluation of texture (Johansen et al. 1991; Chamberlain et al. 1993). Reproducibility of texture measurements is affected by the sampling technique due to heterogeneity of the fillets (Nesvadba et al. 2004). Therefore, it is difficult to find a representative average sample in fish and measurements of textural properties may depend on the location within the fillet. Some authors recommend raw fish to be tested in the form of a fillet or a part of a fillet (Stejskal et al. 2011). The aim of the

Address for correspondence:

František Vácha

University of South Bohemia

Faculty of Fisheries and Protection of Waters, Institute of Aquaculture

Husova tř. 458/102, 37005 České Budějovice, Czech Republic 
study was to evaluate the differences in texture profile of tench flesh under two different ways of fish rearing and determine the effect of sex.

Fish and diets

\section{Materials and Method}

There were two groups of fish used in this study. Fish in the group of extensive culture (EC) were initially pre-reared in a flow-through earth pond. Fish were raised in polyculture with common carp; stocking density for common carp was $500 \mathrm{pcs} \cdot \mathrm{ha}^{-1}$ and for tench $400 \mathrm{pcs} \cdot \mathrm{ha}^{-1}$. Natural production was $220 \mathrm{~kg} \cdot \mathrm{ha}^{-1}$. Oxygen concentration was from 6 to $8 \mathrm{mg} \mathrm{O} \cdot \cdot^{-1}$ without the use of aerator. The $\mathrm{pH}$ level ranged from 6.8 to 7.5 . No supplemental feeding was used.

Fish in the group of intensive culture (IC) were progressively trained to accept the formulated feed. The fish were held in fibreglass tanks connected to a recirculation system. Fish had been well adapted to the experimental diets and environment for more than seven months, as they were previously used in growth trials, during which they were fed the same diets and kept under the same conditions as in the present study. The rearing system consisted of tanks (600 litres of usable volume), mechanical drum filter and three percolating filters. Water temperature was stabilized at $23.3 \pm 0.5^{\circ} \mathrm{C}$, monitored by a thermometer Testo 106 , range -50 to $+275^{\circ} \mathrm{C}$ (Testo Ltd., Czech Republic). The water was oxygenated by oxygen diffusers. The oxygen concentration at the inflow did not fall below $8.0 \mathrm{mg} \mathrm{O} \cdot \mathrm{l}^{-1}$. The total ammonia nitrogen concentration at the inflow and outflow did not exceed 0.29 and $0.68 \mathrm{mg} \mathrm{TAN} \cdot \mathrm{l}^{-1}$, respectively. The water $\mathrm{pH}$ level ranged from 6.85 to 7.14 and it was monitored using a pH Tester 20, range 1.00 to 14.00 (Chromservis Ltd., Czech Republic). The water flow rate was kept constant during the experiment. Fish of this group were fed a commercial diet consisting of $92 \%$ dry matter, $26 \%$ protein, $5.3 \%$ fat, $2.7 \%$ fiber and $4 \%$ ash, determined following the Czech reference methods.

For the trial, the fish size between groups was well balanced with a live weight of $150 \pm 20 \mathrm{~g}$, total body length was $200 \pm 20 \mathrm{~mm}$, body height was $45 \pm 10 \mathrm{~mm}$. The fish were killed, gutted and filleted. For further texture analyses, one hour after killing specific parts of fillet were determined by the position above the lateral line towards the cranial body part in an identical manner (Fig. 1).

Position of sample extraction for texture profile analysis (TPA)

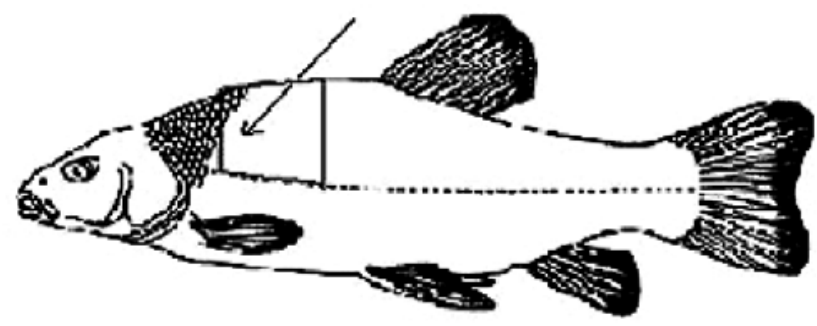

Fig. 1. Illustrative location for sampling in texture profile analysis in the tench body

\section{Texture analyses}

The texture of tench flesh was characterised by the instrumental method texture profile analysis (TPA) for hardness, springiness, cohesiveness, gumminess and chewiness. Samples of raw skinned fillets $24 \mathrm{~mm}$ in diameter were prepared from every tench and stored on ice until analysis. Texture profile analysis was conducted using a Texture Analyser TA.XTPlus (Stable Micro Systems, Godalming, England) with a load cell of $50 \mathrm{~kg}$ at a stable temperature of $17{ }^{\circ} \mathrm{C}$. For texture measurement a compression plate suitable for fish fillets was used. This instrument provides a rigid framework for tension, compression cycling and texture tests to generate true 3-dimensional product analysis of force, distance and time.

\section{Compression plate}

A compression plate of $75 \mathrm{~mm}$ diameter was selected. Penetration depth of $5 \mathrm{~mm}$ into the fillet was selected as the maximum distance which could be applied without breaking the muscle fibres and affecting the muscle structure by disrupting it and leaving a mark on the fillet. Double compression was applied to construct the TPA variables. Then the plate was pressed on the fillet a second time and TPA was obtained by analyzing the forcetime curve (Bourne 1982). The plate was pressed down onto the fillet at a speed of $2 \mathrm{~mm} \cdot \mathrm{s}^{-1}$ until the fillet was compressed to $50 \%$ of its original thickness. Samples were allowed to rebound for $15 \mathrm{~s}$ with the compression plate just contacting the surface. Data collection and calculations (hardness, springiness, cohesiveness, gumminess 
and chewiness) were carried out using the Texture Expert program, version 1.11 (Stable Micro Systems Ltd., Godalming, England). Hardness was defined as the maximum force detected during first compression, expressed in N. Springiness was defined as the ratio of the time or distance from the start of the second area to the second probe reversal over the distance, or the time between the start of the first area and the first probe reversal (Alvarez et al. 2002). Cohesiveness was measured as the ratio of the positive force during the second compression to the positive force during the first compression (Alvarez et al. 2002). Gumminess was defined as the product of hardness $\times$ cohesiveness. Chewiness was defined as hardness $\times$ cohesiveness $\times$ springiness. Cohesiveness, gumminess, springiness and chewiness are dimensionless.

\section{Statistical analysis}

The TPA effect was determined using one-way ANOVA by analysis of variance and Tukey's mean test $(P<0.05)$. The software used was StatSoft, Inc. (2001), STATISTICA CZ, version 6. Student's $t$-test was applied in order to test for the differences between extensive and intensive culture.

\section{Results}

Difference of texture quality (hardness, springiness, cohesiveness, gumminess and chewiness) was studied between fish from extensive and intensive culture. The results of texture analyses in fish from the extensive group were $16.01 \pm 1.91 \mathrm{~N}, 0.72 \pm 0.52,0.66 \pm$ $0.01,10.73 \pm 1.44$ and $7.69 \pm 0.85$ for mean hardness, mean springiness, mean cohesiveness, mean gumminess and mean chewiness, respectively. The results of texture analysis in fish from the intensive group were $15.16 \pm 4.56 \mathrm{~N}, 0.59 \pm 0.06,0.52 \pm 0.04,8.06 \pm 1.91$ and $4.96 \pm 1.73$ for mean hardness, mean springiness, mean cohesiveness, mean gumminess and mean chewiness, respectively. Significant difference $(P<0.05)$ between groups $(\mathrm{EC} / \mathrm{IC})$ was found for hardness, springiness and gumminess; and significant difference $(P<0.01)$ for cohesiveness and chewiness. The results of texture analysis proved, that the flesh of fish from EC group fed natural food was harder (Fig. 2), springier, more cohesive, gummier and chewier compared to IC group.

Flesh of male tench in both groups was harder, more gummy and chewy, compared to female. Springiness was lower in male tench from intensive culture and cohesiveness lower in females from both groups (Table 1).

\section{Discussion}

The experiment proved that the texture of tench flesh can be influenced both by the method of fish culture (extensive, intensive) and by the sex of the fish, as partly mentioned

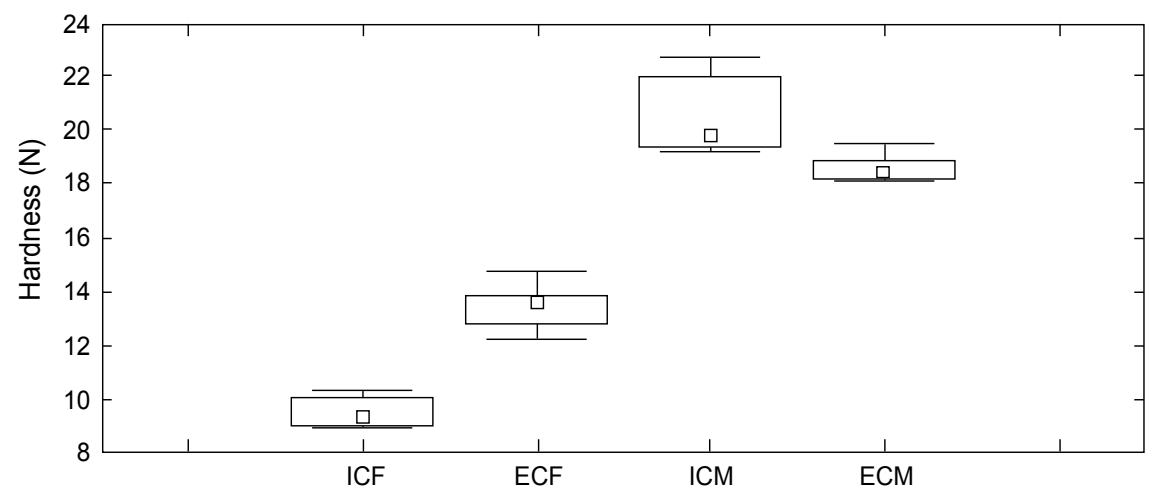

Fig. 2. Statistical evaluation of hardness (in $\mathrm{N}$ ) in tench fillets depending on type of fish culture and sex. ICF - intensive culture female, ECF - extensive culture female, ICM - intensive culture male, ECM - extensive culture male. Data are expressed as mean \pm standard deviation. 
Table 1. Texture profile analyses in tench fillets depending on type of fish culture and sex.

\begin{tabular}{lcccc}
\hline & \multicolumn{2}{c}{ Extensive culture } & \multicolumn{2}{c}{ Intensive culture } \\
\cline { 2 - 5 } Attribute & \multicolumn{1}{c}{ Female } & \multicolumn{1}{c}{ Male } & Female & Male \\
\hline Hardness $(\mathrm{N})^{*}$ & $13.46 \pm 1.05^{\mathrm{b}}$ & $18.55 \pm 0.65^{\mathrm{c}}$ & $9.49 \pm 0.6^{\mathrm{a}}$ & $20.83 \pm 1.31^{\mathrm{c}}$ \\
Springiness & $0.70 \pm 0.07^{\mathrm{b}}$ & $0.75 \pm 0.03^{\mathrm{c}}$ & $0.67 \pm 0.01^{\mathrm{b}}$ & $0.51 \pm 0.04^{\mathrm{a}}$ \\
Cohesiveness & $0.67 \pm 0.01^{\mathrm{c}}$ & $0.66 \pm 0.05^{\mathrm{c}}$ & $0.47 \pm 0.02^{\mathrm{a}}$ & $0.58 \pm 0.02^{\mathrm{b}}$ \\
Gumminess & $8.86 \pm 1.33^{\mathrm{b}}$ & $12.60 \pm 0.44^{\mathrm{d}}$ & $5.50 \pm 0.53^{\mathrm{a}}$ & $10.16 \pm 0.82^{\mathrm{c}}$ \\
Chewiness & $6.60 \pm 0.70^{\mathrm{b}}$ & $8.77 \pm 0.81^{\mathrm{c}}$ & $2.85 \pm 0.26^{\mathrm{a}}$ & $7.14 \pm 0.45^{\mathrm{b}}$ \\
\hline
\end{tabular}

Data are expressed as mean \pm standard deviation. Values in the same row with different superscripts are significant at $P<0.05,(\mathrm{n}=6)$

in allometric analysis published by Jobling (2001). The results also confirmed an important factor in quality assessment of the texture profile; namely myosin, as stated in Ingolfsdóttir (1997), which may influence the hardness level in extensively cultured fish.

Texture quality measurement expressed in particular curves as mentioned by Bourne and Szczesniak (2003) and Cardoso et al. (2009), is used in sensory evaluation and correlates well with the gained data in differences of type of fish culture.

Similar indications were found by the procedure from Rahman (2006) and Bourne (1982), using a double compression technique to perform texture profile analysis in forcetime curves. The technique of measurement is an important factor in fish texture evaluation because of heterogeneity of the fillets, mentioned especially for freshwater fish species as common carp, tench, perch and others by several authors, e.g. Reid and Durance (1992), Nesvadba et al. (2004).

Thus, the experimental protocols already developed can also help in finding more detailed procedure and solutions in the development of fish processing. Texture profile analyses of the tench tend to commercial use where the data can be used in several ways of products development to be incorporated into new fish products. It has a substantial effect on the desirable storage period of frozen fish maintained in proper conditions during their shelf life as mentioned in Rahman (2006).

As the available literature does not refer to particular characteristics included in the analysis of texture profile, this study contributes to more complex investigation into the subject of freshwater fish texture.

\section{Acknowledgements}

The study was supported by the South Bohemian Research Center of Aquaculture and Biodiversity of Hydrocenoses, Grant No. CENAKVA CZ.1.05/2.1.00/01.0024 and CENAKVA II (The results of the project LO1205 were obtained with a financial support from the MEYS of the CR under the NPU I program) and through projects GA JU 074/2013/Z (University of South Bohemia).

\section{References}

Alvarez MD, Canet W, López ME 2002: Influence of deformation rate and degree of compression on textural parameters of potato and apple tissues in texture profile analysis. Eur Food Res Technol 215: 13-20

Bourne MC 1982: Food texture and viscosity: concept and measurement. Academic Press, New York, pp. $45-117$

Bourne MC, Szczesniak AS 2003: Sensory evaluation-texture. In: Caballero B, Trugo L, Finglas P (Eds.): Encyclopedia of food sciences and nutrition, Academic Press, Amsterdam, the Netherlands, pp. 5167-5174

Cardoso CML, Mendes R, Nunes ML 2009: Instrumental texture and sensory characteristics of cod Frankfurter sausages. Int J Food Prop 12: 625-643

Chamberlain AI, Kow F, Balasubramaniam E 1993: Instrumental method for measuring texture of fish. Food Aust 45: 439-433

Ingolfsdóttir S 1997: Post mortem changes in fish muscle proteins structural changes. In: Olafsdóttir G, Luten J, Dalgaard P, Carech M, Verrez-Bagnis V, Martinsdóttir E, Heia K (Eds.): Methods to determine the freshness 
of fish in research and industry. Proceedings of the final meeting of the concerted action "Evalution of fish freshness". 1997 Nov 12-14; Nantes, France. Paris: Intern Inst of Refrigeration, pp. 198-202

Jobling M 2001: Nutrient partitioning and the influence of feed composition on body composition. In: Houlihan D, Boujard T, Jobling M (Eds.): Food Intake in Fish. Blackwell Science, pp. 354-375

Johansen S, Magnussen OM, Nortvedt TS 1991: Bløt Fisk. Norway, Sintef Raport no. STF11 A91075

Johnston IA, Alderson R, Sandham C, Dingwall A, Mitchell D, Selkirk C, Nickell D, Baker R, Robertson B, Whyte D, Springate J 2000: Muscle fibre density in relation to the colour and texture of smoked Atlantic salmon (Salmo salar L.). Aquaculture 189: 335-349

Křížek M, Vácha F, Vejsada P, Pelikánová T 2011: Formation of biogenic amines in fillets and minced flesh of three freshwater fish species stored at $3^{\circ} \mathrm{C}$ and $15^{\circ} \mathrm{C}$. Acta Vet Brno 80: $365-372$

Nesvadba P, Houska M, Wolf W, Gekas V, Jarvis D, Sadd PA, Johns AI 2004: Database of physical properties of agro-food materials. J Food Eng 61: 497-503

Periago MJ, Ayala MD, López-Albors O, Abdel I, Martínez C, García-Alcázar A, Ros G, Gil F 2005: Muscle cellularity and flesh quality of wild and farmed sea bass, Dicentrarchus labrax L. Aquaculture 249: 175-188

Popelka P, Luptáková O, Marcinčák S, Nagy J, Mesarošová L, Nagyová A 2012: The effect of glaze and storage temperature on the quality of frozen mackerel fillets. Acta Vet Brno 81: 397-402

Rahman MS 2006: State diagram of foods: Its potential use in food processing and product stability. Trends Food Sci Technol 17: 129-141

Reid RA, Durance TD 1992: Textural changes of canned Chum salmon related to sexual maturity. J Food Sci 57: $1340-1342$

Stejskal V, Vejsada P, Cepák M, Špička M, Vácha F, Kouřil J, Policar T 2011: Sensory and textural attributes and fatty acid profiles of fillets of extensively and intensively farmed Eurasian perch (Perca fluviatilis L.). Food Chem 129: 1054-1059

Vácha F, Vejsada P, Hůda J, Hartvich P 2007: Influence of supplemental cereal feeding on the content and structure of fatty acids during long-lasting storage of common carp (Cyprinus carpio). Aquacult Int 15: 321-329 